\title{
Toward multi-focal spot remote focusing two-photon microscopy for high speed imaging
}

\author{
Bei $\mathrm{Li}^{*}{ }^{\mathrm{a}}$, Alexander D. Corbett ${ }^{\mathrm{b}}$, Ee Chong ${ }^{\mathrm{a}}$, Edward Mann ${ }^{\mathrm{c}}$, Tony Wilson ${ }^{\mathrm{a}}$, Martin J. Booth ${ }^{\mathrm{a}}$ and \\ Gil Bub ${ }^{\mathrm{d}}$ \\ ${ }^{\mathrm{a}}$ Department of Engineering Science, University of Oxford; ${ }^{\mathrm{b}}$ Department of Physics and Astronomy, \\ University of Exeter; ${ }^{\mathrm{c} D e p a r t m e n t}$ of Physiology, Anatomy and Genetics, University of Oxford; \\ ${ }^{\mathrm{d}}$ Department of Physiology, McGill University
}

\begin{abstract}
Optical sectioning techniques using two-photon excitation of fluorescent indicators are central to diverse imaging applications. The limitations of the technique are low speed and undesirable specimen agitation. In our design, highspeed axial scanning is carried out by moving a reference objective to axially displace the focal spot without introducing significant spherical aberration and any agitation of the specimen. Further, the system is configured to allow switching between single spot and multiple focal spot remote focusing to allow either high dynamic range or high speed imaging.
\end{abstract}

Keywords: two-photon excitation, remote focusing, temporal pixel multiplexing

\section{INTRODUCTION}

Visualizing activity in cardiac and neuronal tissue is a fundamental challenge for biomedicine, as each brief electrical signal propagates rapidly over large distances [1-4]. For example, a single heartbeat involves choreographed waves of electrical activity pervading every cell in the organ. During the same time period, electrical signals in the nervous system can be used to detect the light reflected from an incoming cricket ball (or baseball), calculate its trajectory, and send the motor commands to make the catch. Therefore, a high-speed imaging system is required to capture these rapid events in electrically excitable tissues.

Traditionally, 3D imaging data is collected by physically changing the distance between the objective lens and specimen. This is problematic for two reasons. First, this process is generally slow and second it can lead to undesirable specimen agitation. Remote focusing is a technique which enables optically sectioned, in-focus images of a specimen to be taken outside of the focal plane $[5,6]$. For this reason, it's an appropriate solution to achieve high speed 3D imaging without any agitation of the specimen. Deformable mirror based remote focusing can achieve low aberration imaging but speed is limited by the mirror settling time [7]. Higher-speed refocusing was recently achieved using a carefully designed electrically tunable lens [8], but at the expense of the numerical aperture.

In this paper, we have developed a fast remote focusing system which uses a reference objective to axially displace the focal spot at $500 \mathrm{~Hz}$ rates. This is a significant development as it allows images of the entire specimen volume to be acquired without the need to move either the specimen or the objective. Moreover, the ability to scan over large depth ranges can be achieved at speeds equivalent to lateral scan speeds, allowing fast events in excitable cell networks to be captured. In addition, we have split the beam into multiple spots which allows for parallelization of acquisition in anticipation of incorporating a newly developed temporal pixel multiplexing (TPM) sensor for simultaneous high-speed, high-resolution imaging [9]. This new imaging platform will be used for recording cellular activity and high spatial resolution imaging of anatomical structure.

\section{EXPERIMENTAL SETUP}

The layout of the optical setup is shown in Figure 1. The laser source used for this two photon microscope was a modelocking Ti:sapphire laser (Spectra-Physics Mai Tai DeepSee) which offers over 2.4W of average power and $350 \mathrm{~nm}$

*bei.li@eng.ox.ac.uk

Three-Dimensional and Multidimensional Microscopy: Image Acquisition and Processing XXIV, edited by

Thomas G. Brown, Carol J. Cogswell, Tony Wilson, Proc. of SPIE Vol. 10070, 1007005

(C) 2017 SPIE $\cdot$ CCC code: $1605-7422 / 17 / \$ 18 \cdot$ doi: $10.1117 / 12.2251214$ 
(690-1040 nm) in useable tuning range. The laser output for this experiment had a wavelength of $800 \mathrm{~nm}$ and a $100 \mathrm{fs}$ pulse duration. The power of the laser beam was controlled by a lambda half plate which was placed before the polarizing beam splitter. After beam power control, a 10x anamorphic telescope composed of two achromatic lenses (L1 and L2: $30 \mathrm{~mm}$ and $300 \mathrm{~mm}$ ) expanded the beam to $12 \mathrm{~mm}$ to fill the full aperture of the SLM (Hamamatsu X10468-02 LCOS-SLM). The beam was incident on the center of the $5 \mathrm{~mm}$ galvo scanner after a $3 \times$ beam reduction (L3 and L4: $300 \mathrm{~mm}$ and $100 \mathrm{~mm}$ achromatic lenses). A scanning telescope (L5 and L6: 200mm and 275mm achromatic lenses) was then used to image the beam onto the back aperture of the first reference objective (OBJ1: Olympus UPLSAPO 40×, $0.95 \mathrm{NA})$. The second reference objective (OBJ2: Olympus UPLSAPO $40 \times, 0.95 \mathrm{NA}$ ) was placed nose to nose with the first one to perform remote focusing. A relay telescope (L7 and L8: 200mm and 275mm achromatic lenses) imaged the back focal plane of the second reference objective onto the back focal plane of the imaging objective (OBJ3: Olympus LUMPlanFLN $40 \times, 0.8 \mathrm{NA}$ water dipping objective). As Olympus lenses are designed to operate with $180 \mathrm{~mm}$ focal length tube lenses, the reference objective (OBJ2) had a magnification of $\mathrm{M}_{1}=40 \times(200 / 180)=44.44$, and the imaging objective $(\mathrm{OBJ} 3)$ had a magnification of $\mathrm{M}_{2}=40 \times(275 / 180)=61.11$. Accounting for the different refractive indices of the immersion media $\left(\mathrm{n}_{1}=1, \mathrm{n}_{2}=1.33\right)$, the magnification between conjugate image planes was then given by $\mathrm{n}_{1} \mathrm{M}_{2} / \mathrm{n}_{2} \mathrm{M}_{1} \approx 1[5][10]$. Finally, the imaging objective tightly focused the pulsed source into the specimen, generating two-photon fluorescence. This setup had two different detection methods. With the filter cube slider (FC1 and FC2) set to a silver mirror and a short-pass dichroic mirror (Semrock FF670-SDi01-25x36), the fluorescence signal could be detected using a photon counting PMT (Hamamatsu H7422P-40). With the filter cube slider (FC1 and FC2) set to a short-pass dichroic mirror (Semrock FF670-SDi01-25x36) and a silver mirror, the fluorescence signal was detected using an EMCCD detector (Andor iXon Ultra 888). Refocusing was carried out by moving OBJ1 axially so that different planes of the intermediate image space were imaged without spherical aberration. As a result, we have constructed a system where refocusing can be carried out remotely, without moving the original object and without introduction of spherical aberrations.

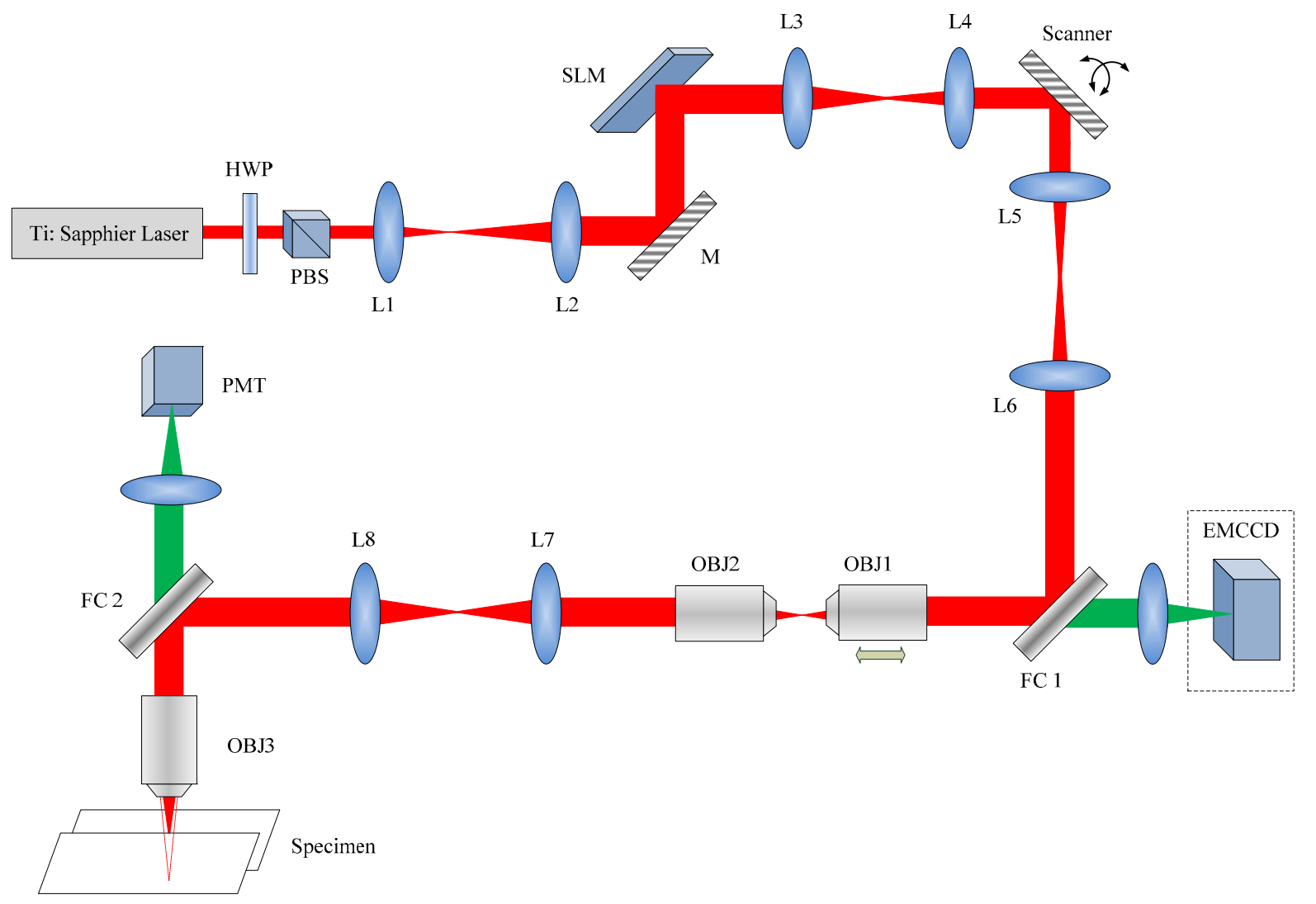

Figure 1. Sketch of the experimental setup. HWP: half wave plate, PBS: polarizing beam splitter, SLM: spatial light modulator, M: silver mirror, L: achromatic lens, Scanner: 2-axis galvo scanner, FC: filter cube slider, F: filter, OBJ: objective, EMCCD: area detector 


\section{RESULTS AND DISCUSSION}

\subsection{PMT detection}

As a first proof-of-principle demonstration of remote focusing on both the PMT and the TPM microscope, we imaged pollen grains (Carolina, Mixed Pollen Grains Slide) using the PMT detection. In this way, the system performed as a standard two-photon microscope with remote focusing. The laser power was adjusted to $5 \mathrm{~mW}$ at the sample (measured using a power meter). The remote focusing is set to $1 \mu \mathrm{m}$ per step which is synchronized with the XY scan. A total of 60 $\mathrm{Z}$ stack images with $512 \times 512$ pixel resolution were acquired using this system as shown in Figure 2 .
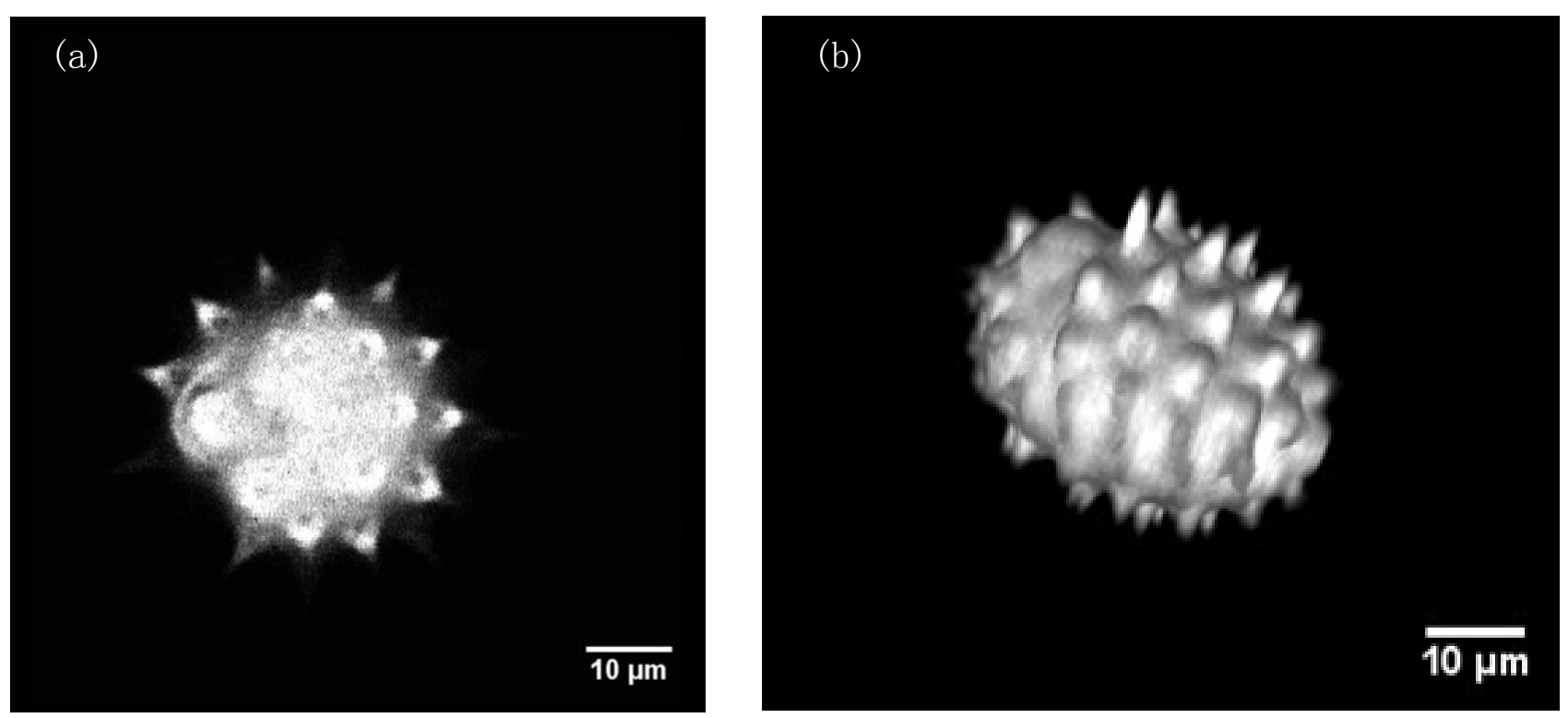

Figure 2. Two photon microscope of pollen grain. (a) Fluorescence image of pollen, (b) rendered 3D image from stacks.

\subsection{EMCCD detection}

For the EMCCD detection experiments, we used the SLM to generate multiple spots in order to increase the whole system acquisition speed [11-13]. The multiple spots were generated via the SLM using the Gerchberg-Saxton algorithm which can precisely position the spots. The use of multiple focal spots is known to reduce image quality due to generation of scattered light. In order to characterize scattered light in our system we illuminated the sample with a single stationary spot and captured fluorescence with the EMCCD (Figure 3a). We found that the majority of captured fluorescence was well localized and rapidly fell of as a function of distance (Figure 3b). We then scanned the spot over the sample and captured fluorescence using a single long EMCCD exposure. Although we expected the image to be blurred, we found that image quality was acceptable and most structures were easily resolved (as was shown in, e.g., ref [11]). We then used the SLM to generate $2 \times 2$ and $4 \times 4$ spots (as shown in Figure 3c), and rescanned the sample (Figures $3 \mathrm{f}$ and $3 \mathrm{~g}$ ). The signal to noise ratio (SNR) was measured for each image by taking a reference image of the same sample using the PMT and the method described in reference [14] (Figure 3d: red, green and blue crosses). The SNR is well approximated assuming a noise contribution due to scattered photons of 0.75 grey levels/pixel (Figure $3 \mathrm{~d}$, grey line). We note that this value is sample dependent, and highly scattering samples may be subjected to higher levels of noise.

The maximum acquisition speed when using a single spot $(512 \times 512$ pixel resolution) is $1.1 \mathrm{~s}$ which was limited by the galvonometer scanner speed. In Figure 3(f) and (g), $2 \times 2$ spots and $4 \times 4$ spots were applied to increase the scanning speed respectively. With same image resolution and image size, the acquisition speed for one frame was reduced to $0.27 \mathrm{~s}$ and 0.06s. Although image quality was degraded as a function of the number of focal spots, this may be an acceptable compromise in some cases. Signal quality will be improved in future generations of the imaging by incorporating a specialized TPM sensor that allows control of pixel exposure times, which will reduce blur by preventing capture of scattered light by out of register pixels. 

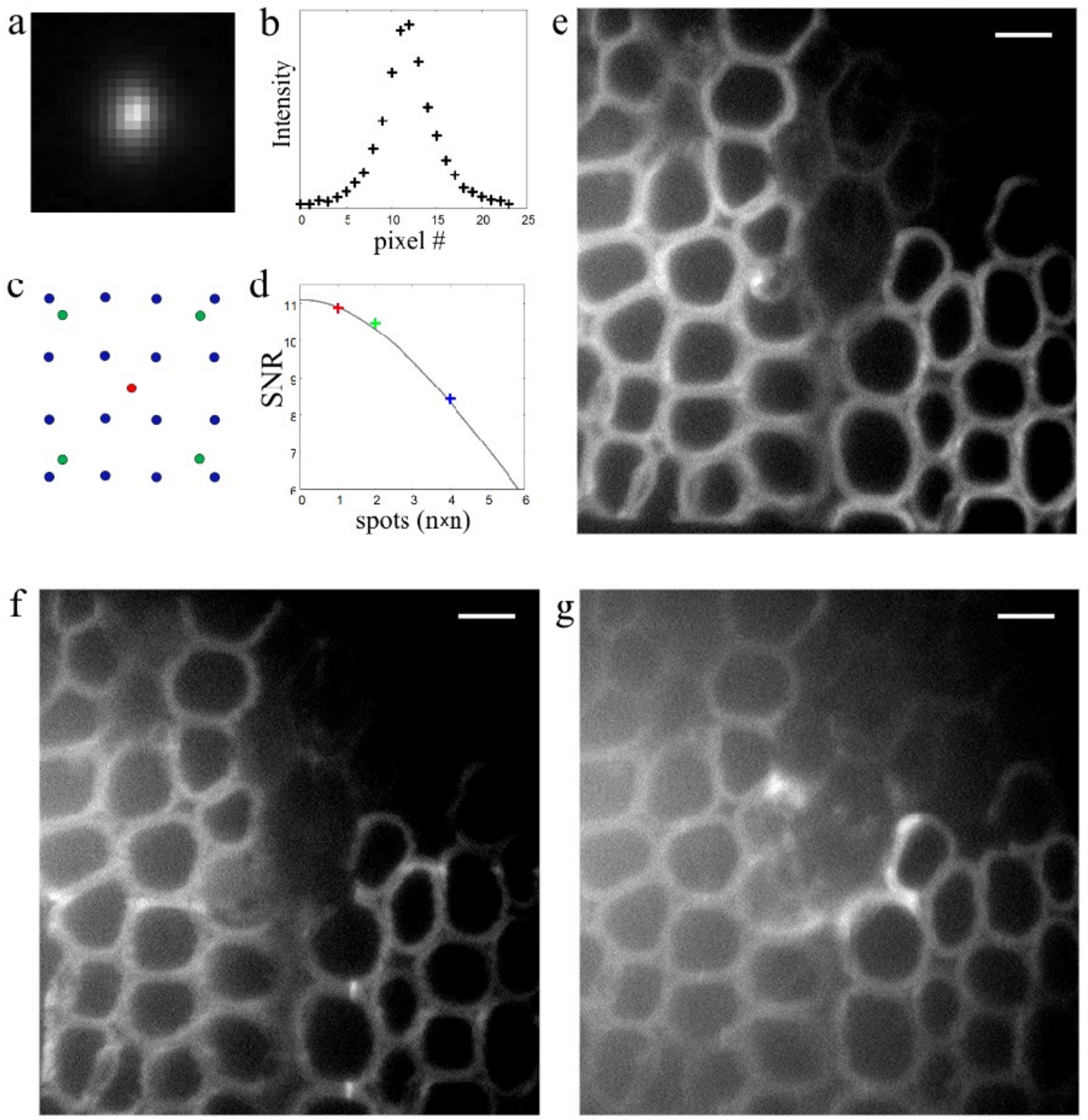

Figure 3. Two photon multi-spot detection of a static scattering sample (Carolina, Pine Stems Slide). (a) illumination with a single illumination spot generates localized fluorescence in addition to significant scatter over 25 pixel radius; (b) intensity of scattered light is highest at the focal spot and falls off as a function of the square of the distance; (c) generated spots after imaging objective (red: single spot, green: $2 \times 2$ spots, blue: $4 \times 4$ spots); (d) estimate of expected (grey line) and measured (red, green and blue crosses) SNR for different dot patterns; (e) two photon fluorescence image obtained using a single spot scan and $1.1 \mathrm{~s}$ frame exposure time; (f) two photon fluorescence image obtained using $2 \times 2$ spot scan and $0.27 \mathrm{~s}$ frame exposure time; $(\mathrm{g})$ two photon fluorescence image obtained using a $4 \times 4$ spot scan and $0.06 \mathrm{~s}$ frame exposure time. (Scale bar: $10 \mu \mathrm{m})$ 


\section{CONCLUSION}

We have developed and demonstrated a remote focusing two-photon microscope with fast axial scanning speed that avoids undesirable specimen agitation. Two detection techniques that offer various trade-offs between temporal and spatial resolution have been implemented on the same microscope to offer a flexible imaging solution.

\section{ACKNOWLEDGEMENTS}

The project is funded by the Medical Research Council through project "MICA: High speed, high resolution imaging of excitable cell networks" (MR/K015877/1).

\section{REFERENCES}

[1] Stosiek, C., Garaschuk, O., Holthoff, K., and Konnerth, A., "In vivo two-photon calcium imaging of neuronal networks," Proc. Natl. Acad. Sci. USA, 100, 7319-7324 (2003).

[2] Botcherby, E. J., Smith, W., Kohl, M., Débarre, D., Booth, M. J., Juškaitis, R., Paulsen, O., and Wilson, T., "Aberration-free three-dimensional multiphoton imaging of neuronal activity at kHz rates," Proc. Natl. Acad. Sci. USA, 109, 2919 - 2924(2012).

[3] Göbel, W., Kampa, B. M., and Helmchen, F., "Imaging cellular network dynamics in three dimensions using fast 3D laser scanning," Nat. Methods, 4, 73-79(2007).

[4] Svoboda, K., and Yasuda, R., "Principles of Two-Photon Excitation Microscopy and Its Applications to Neuroscience," Neuron, 50(6), 823-839(2006).

[5] Botcherby, E. J., Juškaitis, R., Booth, M. J., and Wilson, T., "An optical technique for remote focusing in microscopy," Optics Communications, 281(4), 880-887(2007).

[6] Botcherby, E. J., Booth, M. J., Juskaitis, R., and Wilson, T., "Real-time extended depth of field microscopy," Opt. Express, 16(26), 21843-8(2008).

[7] Zurauskas, M., Frade, M., and Booth, M. J., "Deformable mirror based remote focusing for fast threedimensional microscopy,” Proc. SPIE 9713, (2016).

[8] Grewe, B. J., Voigt, F. F., Hoff, M., and Helmchen, F., "Fast two-layer two-photon imaging of neuronal cell populations using an electrically tunable lens," Biomed. Opt. Express, 2(7), 2035-2046 (2011).

[9] Bub, G., Tecza, M., Helmes, M., Lee, P., and Kohl, P., "Temporal pixel multiplexing for simultaneous highspeed, high-resolution imaging," Nat. Methods, 7(3), 209-11(2010).

[10] Corbett, A., Burton, R., Bub, G., Salter, P., Tuohy, S., Booth, M.J., and Wilson, T., "Quantifying distortions in two-photon remote focussing microscope images using a volumetric calibration specimen." Front. Physiol., 5(384), (2014).

[11] Sacconi, L., Froner, E., Antolini, R., Taghizadeh, M. R., Choudry, A., and Pavone, F.S., "Multiphoton multifocal microscopy exploiting a diffractive optical element," Optics Lett., 28(20), 1918-20(2003).

[12] Niesner, R., Andresen, V., Neumann, J., Spiecker, H., and Gunzer, M., "The power of single and multibeam two-photon microscopy for high-resolution and high-speed deep tissue and intravital imaging," Biophys. J., 93(7), 2519-2529(2006).

[13] Pozzi, P., Gandolfi, D., Tognolina, M., Chirico, G., Mapelli, J., and D’Angelo, E., "High-throughput spatial light modulation two-photonmicroscopy for fast functional imaging," Neurophotonics, 2(1), 015005 (2015).

[14] Gonzalez R. C., and Woods R. E., "Digital Image Processing," Third Edition, Prentice Hall2008. ISBN: 013168728 (2008). 